\title{
A Look at the Security and Privacy of Fitbit as a Health Activity Tracker
}

\author{
Jason Orlosky \\ Osaka University and Augusta University \\ orlosky[at]lab.ime.cmc.osaka-u.ac.jp \\ Heather Yates \\ Augusta University \\ hyates[at]augusta.edu
}

\author{
Onyeka Ezenwoye \\ Augusta University \\ oezenwoye[at]augusta.edu \\ Gina Besenyi \\ Kansas State University and Augusta University \\ gbesenyi[at]ksu.edu
}

\begin{abstract}
Given the popularity of consumer grade wearable health trackers, there is an increasing need to evaluate their accuracy and security. In this paper, we present the results of a study with 24 participants who used and evaluated a small form factor personal health device, the Fitbit Blaze. Our study includes both the analysis of data taken from an exercise-based experiment and a review of the security risks associated with current protocols used to access Fitbit device data and participant information. In addition to discussion of the FitBit's accelerometer and pulse data as compared to clinical grade devices, we gathered and analyzed subjective participant data on usability and perception of privacy and security using both quantitative and subjective methods. Results showed that FitBit accuracy was not equivalent to medical grade devices, that a majority of risk comes from potentially fraudulent third party applications, and that users are typically justified in their concerns.
\end{abstract}

\section{CCS CONCEPTS}

- Computer-Communication Networks $\rightarrow$ General.

\section{KEYWORDS}

Health tracker, privacy, security, perception, validation

\section{ACM Reference Format:}

Jason Orlosky, Onyeka Ezenwoye, Heather Yates, and Gina Besenyi. 2019. A Look at the Security and Privacy of Fitbit as a Health Activity Tracker. In 2019 ACM Southeast Conference (ACMSE 2019), April 18-20, 2019, Kennesaw, GA, USA. ACM, New York, NY, USA, 4 pages. https://doi.org/10.1145/3299815. 3314468

\section{INTRODUCTION}

Personal health monitoring devices have recently made their way into consumer markets as a popular way to self-monitor physical activity and other health behaviors. One of the most popular formfactors for these devices is a wrist-worn activity tracker, which

Permission to make digital or hard copies of all or part of this work for personal or classroom use is granted without fee provided that copies are not made or distributed for profit or commercial advantage and that copies bear this notice and the full citation on the first page. Copyrights for components of this work owned by others than ACM must be honored. Abstracting with credit is permitted. To copy otherwise, or republish, to post on servers or to redistribute to lists, requires prior specific permission and/or a fee. Request permissions from permissions@acm.org.

ACMSE 2019, April 18-20, 2019, Kennesaw, GA, USA

(C) 2019 Association for Computing Machinery.

ACM ISBN 978-1-4503-6251-1/19/04 . .\$15.00

https://doi.org/10.1145/3299815.3314468 typically stays with a user throughout the day. These devices can be beneficial for improving physical activity for different user groups.

However, like many other tracking technologies, wearable devices carry a significant amount of user data, some of which potentially carries information about the person's genetic factors and disease state. For example, Farzanehfar et al. showed that motion trackers can be used to infer and monitor disease state, such as Parkinson's disease [7], and can even be used to diagnose tremor [18]. This is significant from a security and privacy perspective, especially concerning the Health Insurance Portability and Accountability Act (HIPAA) rules and regulations. Unlike data that is typically recorded and confined to a lab or hospital setting or transferred over physical media [12], Fitbit devices leave secured areas with personal data and travel with the user into many domains.

To help explore the issue of security and determine user perceptions of personal data and privacy, this paper 1) analyzes the authentication technologies associated with wearable tracking devices, 2) describes the results of a series of experiments testing accuracy of information of wearable trackers against two different devices, as shown in Figure 1, with a group of participants in different exercise scenarios, and 3) describes detailed results of subjective evaluations using both Likert scale scoring and discussion groups.

\section{PRIOR WORK}

Prior work typically falls into two categories, including 1) the evaluation of security and privacy management technology and mechanisms, and 2) user perceptions of the resulting privacy, security, and data access.

\subsection{Wearable Device Security}

Some research such as that by [5], looked at the Bluetooth and network traffic of the FitBit flex, and uncovered several vulnerabilities such as static MAC adressing and exposed credentials during pairing. Work by Fereidooni et al. and Rahman et al. also describe security analysis of the technical aspects of the protocols for data collection and transmission across Fitbit devices, mobile applications, and Fitbit web services [8], [13]. They found some security vulnerabilities pertaining to how user data is collected and transmitted across the Fitbit ecosystem. Rieck more recently showed that out-of-date firmware can also be a source of vulnerability in wearable devices [14].

These works are different from ours in that they do not look at the security and privacy issues concerning how Fitbit user data is accessed by third-party applications. Whereas some data, like 
anonymized demographics, are not typically an issue, the amount of exercise a particular user accomplishes in a day can be tied to insurance premiums, influence that person's work life, become the target of litigation, or have other unintended influence.

\subsection{Perceptions of Privacy and Security}

A second related area that has been well studied is that of privacy and security with respect to user perceptions [10]. Various types of devices come with different perceived and actual risks for endusers. One study that examines such perceptions of electronic data was that of Dinev et al. [6]. This study looked into both perceived control and trust of data, and found that perceived effectiveness of technology privacy mechanisms showed significant positive effect on both perceived privacy control and trust. In some sense, technology privacy mechanisms are the equivalent of security in that they establish control over personal data. Subedar El-Khatib made the argument that users are essentially posed with a trade-off between data privacy and security, since monitoring and maintaining one's own health may take precedence over the desire for security [16]. Marakhimov et al. looked even deeper into this concern by observing how consumers attained extended use of healthcare wearable devices despite the presence of health and privacy concerns [11]. The study identifies two coping mechanisms, problemand emotion- based coping, which were concluded to have a direct effect on the duration of use. In other words, the better the ability to cope with stress or discord resulting from concerns, the longer a user would be willing to use the technology.

In contrast with these studies, we obtained subjective data directly from users in our physical activity study. Each participant gave us control of his or her data, meaning the perceptions listed on our questionnaires were directly influenced by the knowledge that anonymized data would be collected and used. Moreover, by analyzing data accuracy in comparison with a commercial grade health tracking device, we can determine whether patient concerns with security or privacy are justified.

\section{ANALYSIS OF PRIVACY AND SECURITY RISKS}

To establish the relationship between security, privacy and user perception, we first needed to explore current security risks in more depth. With wearable computers becoming ubiquitous, it is important to assess the security threats to personal information that these devices pose [1]. Security can be viewed as the ability of a system to prevent unauthorized access or use of any aspect of the system, including data [3]. Privacy is an attribute of data that specifies its visibility and is enforced through security. Private data has restricted visibility as opposed to public data.

Typically, users of devices such as fitness trackers are not aware of the security implications of such devices, and can reveal sensitive or secure information via aggregate or position data. Fitbit user data is stored as part of the user's Fitbit account profile.

\subsection{Third-party Applications}

One type of risk that is often overlooked is the fact that outside applications are often given access to user data. In order for a thirdparty application to access Fitbit user device data, permission is

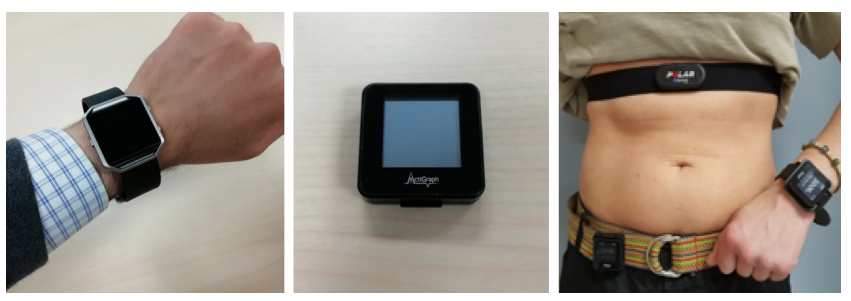

Figure 1: Images of the Three Devices used During Experimentation. From left to right: Fitbit Blaze (Non-dominant Wrist), Actigraph GTX9 Accelerometer (Non-dominant Wrist), and a Polar Heart Rate Monitor (chest)

required from the device user. Fitbit have instituted mechanisms for attaining this authorization from the user. A third-party application that needs to access user data, first needs to be registered with Fitbit. The application then needs to implement a Fitbit data access protocol to request permission from the Fitbit user to access their data. If access permission is granted, the application can both read and write to the device data that is stored as part of the device user's Fitbit account.

Third-party applications can then request permission to access scopes of user data. At the time of granting access to their data, the Fitbit user can determine what data scopes to allow access to. Available data scopes include the activity scope, which contains data on steps taken, distance covered, calories burned and activity minutes. Other scopes include sleep logs, heart rate, weight, GPS location, nutrition, and friends list.

The ability to modify user data does raise some security concerns as an application with access to user data could maliciously or inadvertently change a user's records. The lack of user education on this part is an additional source of risk with respect to third party applications. For example, using Facebook or Google accounts as a $\log$ in can result in data breaches if accounts are hacked. Currently, no mechanism exists for a user to grant read access but not write access to their data.

\subsection{OAuth 2.0}

The Fitbit data access protocol leverages the OAuth 2.0 [9] authentication protocol. OAuth is a user authentication standard whereby permission to a user's account data only needs to be granted once to a third-party application. After permission is granted, the thirdparty application can access user data until permission is revoked by the user. Fitbit users can revoke data permissions via their Fitbit mobile application account settings. Fitbit supports two authorization modes for access to user data, namely, the Authorization Code Grant and Implicit Grant. For Authorization Code Grant, any permission, once granted, does not expire and will not need re-authorization.

This open-ended authentication model does present some security and privacy concerns for users' account data as over time, the user is likely to forget which applications have access to their data. Also, mechanisms do not exist for the user to review or revoke access permissions directly on the Fitbit Blaze. For the Implicit Grant, permissions have a lifetime, and lifetime options are, one day, one week, and thirty days. Upon expiration of permission, a user would have to re-authorized access to their account data. Third-party applications have a choice of either authorization modes. 
OAuth 2.0 does have some known security vulnerabilities [9], the primary vulnerability being phishing attacks. In a phishing attack, a malicious application could pose as a legitimate thirdparty application and redirect a Fitbit user to a website that aims to obtain their login credentials. Fitbit's use of the OAuth 2.0 protocol exposes users to this phishing attack vulnerability.

\section{EXPERIMENTS}

To gather quantitative and perceptual data, we conducted a short experiment based on the design by Sushames et al. [17]. Our experiment was within-subjects, including 24 participants (13 male) with an average age of 25.73 (stdev 3.61). Participants were screened to ensure low cardiovascular risk, a body mass index (BMI) under 29, and the ability to complete physical activity on a treadmill. As a way to prevent exposure of the participants to additional security risks, experimenters guided participants through the setup of a dummy Fitbit account by entering the participant's height, weight, sex, and stride length. This dummy account was used for all participants so that data was stored in a central location. Staff then assisted participants in syncing their account to a Fitbit Blaze device provided to them for purposes of the study. Participants wore the Fitbit (wrist), an Actigraph GTX9 accelerometer (right hip), and a Polar heart rate monitor (chest) for data validation.

Participants completed four, six-minute physical activity bouts with four minute rest periods in a controlled lab setting. Activities included: treadmill walking, treadmill incline walking, treadmill jogging, and stair stepping for a total of 24 minutes of activity. Physical activity intensities were participant-determined using Borg's Rate of Perceived Exertion (RPE) ranging from level four (light to moderate) during walking to seven (vigorous) during stair stepping. Activity protocol was repeated for two exercise sessions several days apart. After exercise bouts were completed, participants were educated about how to access and review their physical activity data through the Fitbit application.

\subsection{Data Collection and Software}

In order to collect data and at the same time protect user privacy, we developed an additional protocol to be able to log data and anonymize personal information. Typical approaches to health tracking devices may use an actual participant's login information and then download information from that account. To prevent the need for this, we created a single account, and granted permission for a single application. Prior to each participant's exercise sessions, we re-entered that participant's height, weight, sex, age and stride length information. This prevented participants from having to log in with a personal login and password, but still allowed us to take advantage of the Fitbit's personalized activity tracking algorithms.

The application we developed let us download data using the Oauth2 protocols mentioned previously. Though users of Fitbit devices are only privy to aggregate information like daily or current steps, developers can access an "intraday" time series that allows for minute to minute data downloads. Using this intraday series, the Fitbit API, and a template of existing code [2] for accessing intraday data, we came up with a custom application to download a single series for multiple days, which included all participant information over the duration of the study. The experiment sessions were also timestamped (manually synchronized with the Fitbit), so all data from the download could be matched to a particular participant and session independently of login information.

Data was aggregated into 60 second epochs and analyzed using intraclass correlations (ICCs) using a two-way random effects model with absolute agreement. Bland-Altman plots further explored each intensity level and aided understanding of limits of agreement. To test robustness, we compared step and heart rate variables from the Fitbit Blaze against data from the Actigraph GTX9 (steps) and Polar chest monitor (heart rate). Reliability analysis compared Fitbit step and heart rate data between sessions one and two across all users. Overall, Fitbit Blaze exhibited poor validity for heart rate (HR) $(0.26)$ and moderate validity for steps $(0.58)$ and displayed moderate reliability for HR (0.63) and good reliability for steps (0.80). Results varied with intensity, with best values during treadmill jogging. While none of the data taken from these wearable monitors can represent a true ground-truth [4], significant variability existed between consumer and medical grade devices.

\subsection{Subjective Data and Scoring}

After participants completed Fitbit validity and reliability testing they were asked to complete qualitative survey data. Despite the challenges with physical security of the device itself, it was essential to determine how users of the device actually perceive the privacy and security issues surrounding health trackers, especially for use in research. To do so, we administered a web based questionnaire with six statements (asked in randomized order) rated from agree to disagree on a 7-point Likert scale. In addition to two entries for age and gender, the subjective items were as follows:

(1) I have privacy concerns regarding the Fitbit device.

(2) I have security concerns regarding the Fitbit device.

(3) I would like to use a Fitbit device to help track my fitness.

(4) I am willing to use a Fitbit device as part of a research study.

(5) I feel that I have adequate control over my Fitbit data.

(6) I feel that access to my Fitbit data should be further restricted.

After the subjective ratings were submitted, we conducted four small focus groups, ranging from 6-9 participants, lasting approximately 45 minutes each. Using a semi-structured focus group guide consisting of open-ended questions and probes one experimenter facilitated discussions concerning the issues in the questionnaire.

\section{RESULTS}

Primary results of the subjective ratings were that security concerns outweighed privacy concerns and that participants were more willing to use the tracker for research than for personal use. A two sample t-test (assuming unequal variances) between the privacy and security concerns revealed a near significant difference ( $t$ Stat $=-1.68, \mathrm{p}<0.05$ ) between the two, highlighted in green in Figure 2. Rating averages were 3.68 for privacy vs. 4.32 for security. We also found a significant difference between the willingness to use the device for personal vs. research reasons, as shown in the middle two bars of Figure 2. T-tests also revealed a significant difference (tStat $=2.53, \mathrm{p}<0.01)$ in this case, with personal use coming in at 3.03 versus research at 3.64 .

Relevant comments from the focus groups indicated that participants were uncomfortable with Fitbit's approach of using social 


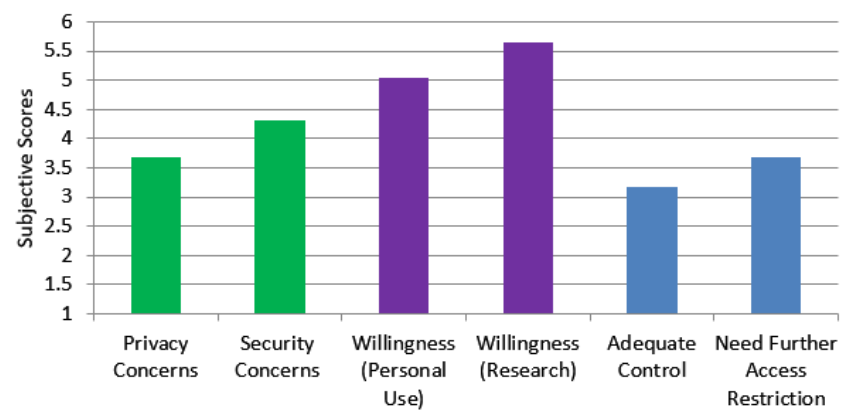

Figure 2: Subjective Differences in Perceived Concerns, Willingness to use Devices, and Data Control

network profiles to identify friends, were unsure how much of their data would be shared with 3rd party services, and were especially concerned about location data breaches and misuse. Participants indicated they would be comfortable sharing Fitbit data with medical and research entities, but commercial application of Fitbit data such as for insurance, advertising, or workplace wellness met with resistance for fear of abuse.

\section{DISCUSSION}

One of the areas we wanted to explore with this study was whether privacy and security concerns are justified for wearable health trackers. To evaluate this question, we analyzed the actual security risks with such devices and solicited feedback from users that participated in a study that was designed to determine the perception of risk as well as confirm the validity of data being produced.

Based on our security analysis, we found that the authorization code grant and implicit grant structures facilitated by the Oauth2 [9] protocol are not only robust, but also give the user adequate control to revoke access as desired. However, despite authentication security, the protocols would not prevent access to data from a bad actor on the application side. As such, it is of the utmost importance that users be educated about what applications they are using and what permissions they are granting in a straight-forward way.

One strategy to mitigate this type of improper access for research purposes is to grant access using substitute accounts. For example, instead of using their personal accounts, users can create an account using a fictitious e-mail address or profile. Subjective ratings indicated that users were more willing to give access to their data for research than for other uses, likely in part due to our procedure of using a non-personal account for data collection. Moreover, data validation showed that accuracy of step and heart data, though correlated, do not meet the same accuracy specifications for a medical grade device. Though aggregate data can still be used to determine high-level health information, a data breach is less of a concern since data exposure is less likely to be exploited by a litigator, insurance company, or entity that may seek to base claims on the validity of health information.

\section{CONCLUSION}

In this paper, we present a study that explores wearable health tracker security, user perceptions of the technology, and accuracy of the device during a physical activity experiment. Results showed that security is more of a concern than privacy, and that actual device accuracy is not on par with a medical grade system. As such, developers should pay attention to preventing unauthorized access and educating users, just as much as improved security algorithms.

Moreover, clinical grade devices that may eventually be manufactured or produced as commodity devices in the future should have additional levels of protection to prevent misuse of user data. Based on the data comparison, there is a marked need for improved accuracy for future commodity devices in order for users to be able to rely on this data for health based evaluation.

\section{ACKNOWLEDGEMENTS}

This work was funded in part by the United States Department of the Navy, Office of Naval Research, Grant \#N62909-18-1-2036. Informed consent was obtained from all individual participants included in the study.

\section{REFERENCES}

[1] M. B. Blake. (2015). Worrying about Wearables, IEEE Internet Computing, 19(5), pp. $4-5$

[2] S. Bromberg. "GoogleFitbit." https://github.com/simonbromberg/googleFitbit/ blob/master/intraday.gs. Accessed November 3rd, 2017.

[3] B. Bruegge and A. H. Dutoit. (2009). Object-Oriented Software Engineering Using UML, Patterns, and Java. Prentice Hall Press, 0136061257.

[4] L. Cadmus-Bertram, R. Gangnon, E. J. Wirkus, K. M. Thraen-Borowski, and J. Gorzelitz-Liebhauser. The Accuracy of Heart Rate Monitoring by Some WristWorn Activity Trackers. Ann Intern Med. 2017;166(8):610-612.

[5] B. Cyr, W. Horn, D. Miao, and M. Specter. (2014). Security Analysis of Wearable Fitness Devices (Fitbit). Massachusetts Institute of Technology.

[6] T. Dinev, V. Albano, H. Xu, A. D'Atri, and P. Hart. (2016). Individuals' Attitudes Towards Electronic Health Records: A Privacy Calculus Perspective. In Advances in Healthcare Informatics and Analytics. pp. 19-50. Springer.

[7] P. Farzanehfar and M. Horne. (2017). Evaluation of the Parkinson's KinetiGraph in Monitoring and Managing Parkinson's Disease. Expert Review of Medical Devices, 14(8), pp. 583-591

[8] H. Fereidooni, J. Classen, T. Spink, P. Patras, M. Miettinen, A. Sadeghi, M. Hollick, and M. Conti. (2017) Breaking Fitness Records Without Moving: Reverse Engineering and Spoofing Fitbit. International Symposium on Research in Attacks, Intrusions, and Defenses.

[9] D. Hardt. "The OAuth 2.0 Authorization Framework" https://tools.ietf.org/html/ rfc6749. Accessed November 3rd, 2017.

[10] G. Kenny and R. Connolly. (2017). Examining Citizens' Health Information Privacy Concerns: An Extension of the IPC Instrument.

[11] A. Marakhimov and J. Joo. (2017). Consumer Adaptation and Infusion of Wearable Devices for Healthcare. Computers in Human Behavior, 76, pp. 135-148

[12] R. Prakash, A. B. Ganesh, and S. Sivabalan. (2017). Network Coded Cooperative Communication in a Real-Time Wireless Hospital Sensor Network. Journal of Medical Systems, 41(5), p. 72

[13] M. Rahman, B. Carbunar, and M. Banik. (2013). Fit and Vulnerable: Attacks and Defenses for a Health Monitoring Device. arXiv preprint arXiv:1304.5672.

[14] J. Rieck. (2016). Attacks on fitness Trackers Revisited: A Case-Study of Unfit Firmware Security. arXiv preprint arXiv:1604.03313.

[15] J. Srinivas, D. Mishra, and S. Mukhopadhyay. (2017). A Mutual Authentication Framework for Wireless Medical Sensor Networks. Journal of Medical Systems, 41(5), 80 .

[16] H. Subedar, and K. El-Khatib. (2015, October). Privacy and Security Concerns for Health Data Collected using Off-the-shelf Health Monitoring Devices. In 2015 IEEE 11th International Conference on Wireless and Mobile Computing, Networking and Communications (WiMob) (pp. 341-348). IEEE.

[17] A. Sushames, A. Edwards, F. Thompson, R. McDermott, and K. Gebel. (2016). Validity and reliability of Fitbit Flex for Step Count, Moderate to Vigorous Physical Activity and Activity Energy Expenditure. PloS one, 11(9), e0161224.

[18] D. J. Wile, R. Ranawaya, and Z. H. Kiss. (2014). Smart Watch Accelerometry for Analysis and Diagnosis of Tremor. Journal of Neuroscience Methods, 230, pp. 1-4

[19] W. Zhou and S. Piramuthu. Security/Privacy of Wearable Fitness Tracking IoT Devices. In Information Systems and Technologies (CISTI), 2014 9th Iberian Conference on (pp. 1-5). IEEE. (2014) 